\title{
A Thermodynamical Model of Liquid-Vapor Interaction
}

\author{
Hala Ghazi ${ }^{1}$, François James ${ }^{2}$, Hélène Mathis ${ }^{1}$ \\ ${ }^{1}$ LMJL, University of Nantes \\ 2 rue de la Houssinière, Nantes, France \\ Hala.Ghazi@univ-nantes.fr; Francois.James@ math.cnrs.fr \\ ${ }^{2}$ MAPMO, University of Orléans \\ Rue de Chartres, Orléans, France \\ Helene.Mathis@univ-nantes.fr
}

\begin{abstract}
In this work, we propose a model of liquid-vapor phase transition allowing the appearance of metastable states contained in the reduced equation of van der Waals. Following the Gibbs formalism, we assume that the entropy of the fluid is described by its mass $\mathrm{M}$, its volume $\mathrm{V}$ and its energy $\mathrm{E}$. Using the second principle of thermodynamics, we construct a maximization problem under constraints of the system entropy. Then, thanks to the Caratheodory's theorem, we get a mathematical proof for the Gibbs phase rule. This approach allows us to recover all states of equilibrium: Maxwell's equilibrium, pure stable and metastable states, by studying the maximization problem constructed above.
\end{abstract}

Keywords: Thermodynamics of Phase Transition, Van Der Waals EOS, Gibbs Phase Rule, Optimisation Problem.

\section{Introduction}

We propose a mathematical formulation for a single liquid or vapor fluid allowing the appearance of all kinds of equilibria. Usually, the strategy is to use a diphasic model where each phase is described by a concave entropy ([1],[2]). From the second principle of thermodynamics, the entropy of the mixture model is the maximum of the total entropy and it corresponds to the concave hull of partial entropies [1]. Here we propose a model as a maximization problem under constraints where each phase is covering by the same non concave entropy like the van der Waals EoS. Using the concepts of convex analysis we prove that the number of phases is limited to 2 . Then, the study of this optimization problem under constraints allows to characterize the equilibria for the thermodynamic system.

\section{Thermodynamics and Optimization Problem}

The goal of this section is to describe the liquid-vapor phase transition from a thermodynamical point of view.

\subsection{Description of a Single Fluid}

We consider a fluid of mass $M \geq 0$, covering a volume $V \geq 0$ and characterized by its energy $E \geq 0$. Using the Gibbs' formalism (see [3], [4]) we define the extensive entropy $\mathrm{S}$ of the fluid as a function of $M, V$ and $E$ :

$$
S:(M, V, E) \rightarrow S(M, V, E)
$$

All the above quantities are said extensive quantities: if the system is doubled, then its mass, volume, energy and entropy also double. In the mathematical sense, any extensive quantity is said positively homogeneous of degree 1(PH1) verifying the following relation:

$$
\forall \lambda>0, S(\lambda M, \lambda V, \lambda E)=\lambda S(M, V, E)
$$

Moreover, we assume without loss of generality that the entropy function $S$ belongs to $C^{2}(\mathbb{R} \times \mathbb{R} \times \mathbb{R})$. It allows to introduce intensive quantities, that are positively homogeneous functions of degree $0(P H 0)$. Thus, we commonly define the pressure $p$, the temperature $T$ and the chemical potential $\mu$ as following [6]: 


$$
\frac{1}{T}=\frac{\partial S}{\partial E}(M, V, E), \quad \frac{p}{T}=\frac{\partial S}{\partial V}(M, V, E), \quad \frac{\mu}{T}=\frac{-\partial S}{\partial M}(M, V, E)
$$

We recall the fact that all these quantities $p, T$ and $\mu$ are defined only when the system is at equilibrium. Furthermore, as $S$ is considered to be a thermodynamic potential then it must be a total differential, so the intensive quantities $p, T$, and $\mu$ must verify the following classical thermodynamic extensive Gibbs' relation:

$$
d S=\frac{-\mu}{T} d M+\frac{P}{T} d V+\frac{1}{T} d E
$$

Since $S$ is a $P H 1$ function, it verifies the Euler relation (see [6]), which gives

$$
S(M, V, E)=\frac{-\mu M}{T}+\frac{p V}{T}+\frac{E}{T}
$$

Dividing the equation (5) by $M$, then we can define the intensive entropy, denoted $s$, for a fixed mass $M>0$ :

$$
s(\tau, e)=\frac{-\mu(\tau, e)}{T(\tau, e)}+\frac{p(\tau, e)}{T(\tau, e)}+\frac{e}{T(\tau, e)} \text { where } \tau=\frac{V}{M}, e=\frac{E}{M}
$$

We then recover the intensive Gibbs relation:

$$
T d s=d e+p d \tau
$$

In this work we are interested to a non necessary concave nor convex entropy function s that verifies

$$
\frac{\partial^{2} s}{\partial e^{2}}<0
$$

An example of such Equation of State $(\mathrm{EoS})$ is the van der Waals EoS with the entropy function

$$
s(\tau, e)=C_{v} \ln (a / \tau+e)+R \ln (\tau-b)+s_{0}
$$

where $R$ is the universal constant of gas, $C_{v}$ the calorific constant at constant volume, $s_{0}$ the entropy of reference, $a$ and $b$ are the two constants of van der Waals EoS depending of each gas [3,5].

\subsection{Description of the Phase Transition}

In this part, we want to describe the the formalism describing a phase transition with an appropriate EoS. We adopt the same approach used in [6]. We consider a fluid presented under I subsystems where each subsystem $i$, for $i=1, \ldots, I$, is characterized by its mass $M_{i} \geq 0$, its volume $V_{i} \geq 0$ and its energy $E_{i} \geq 0$. Moreover, we assume that each subsystem $i$ follows the same non concave entropy $S\left(M_{i}, V_{i}, E_{i}\right)$. In our work, we always preserve the principles of mass conservation, and the conservation of energy. Then the mass and the energy of the complete system are:

$$
M=\sum_{i=1}^{I} M_{i}, \quad E=\sum_{i=1}^{I} E_{i}
$$


Furthermore, we suppose that all the subsystems are immiscible, so that the total volume is

$$
V=\sum_{i=1}^{I} V_{i}
$$

Out of thermodynamic equilibrium, the entropy of the system is the sum of the partial entropies of each subsystem:

$$
S\left(\left(M_{i}, V_{i}, E_{i}\right)_{i}\right)=\sum_{i=1}^{I} S\left(M_{i}, V_{i}, E_{i}\right)
$$

For fixed mass $M$, volume $V$ and energy $E$, the thermodynamic equilibrium states is the maximum of the entropy mixture according to the second principle of thermodynamics. This corresponds to determine the solutions of the following maximization problem:

$$
\sup \left\{\sum_{i=1}^{I} S\left(M_{i}, V_{i}, E_{i}\right) ; M_{i} \geq 0, V_{i} \geq 0, E_{i}, \geq 0\right\}
$$

under the constraints

$$
\sum_{i=1}^{I} M_{i}=M, \sum_{i=1}^{I} V_{i}=V, \sum_{i=1}^{I} E_{i}=E
$$

We denote that $I$ is also a parameter of the optimisation problem under constraints (13)-(14).

In the sequel, we denote $\varphi_{i}=M_{i} / M \in[0,1]$ the mass fraction, $\tau_{i}=V_{i} / M_{i} \geq 0$ the specific volume and the specific energy of the phase $i=1, \ldots I$. Then the maximization problem (13) can be written as

$$
\sup \left\{\sum_{i=1}^{I} \varphi_{i} s\left(\tau_{i}, e_{i}\right) ; \quad \tau_{i} \geq 0, \quad \varphi_{i} \geq 0, e_{i} \geq 0\right\}
$$

under the constraints

$$
\sum_{i=1}^{I} \varphi_{i}=1, \quad \sum_{i=1}^{I} \varphi_{i} \tau_{i}=\tau \quad \sum_{i=1}^{I} \varphi_{i} e_{i}=e
$$

\section{Phase Rule and Equilibrium States}

The aim of this section is to show the maximal number of phases which can be reached at the thermodynamic equilibrium and then to compare this result with the physical rule namely the Gibbs phase rule. The last step is to describe the equilibria of the thermodynamic system by analysing the previous maximization problem.

\subsection{The Phase Rule}

We recall that $I$ is an unknown of the maximization problem (13)-(14), and that indicates the number of phases potentially present at equilibrium. So in order to know the value of the number $I$,we refer to some concepts of convex analysis, in particular according to the Caratheodory's theorem (please see [7], [8] for detailed proofs), we have $I \leq 3$. This means that at the most three phases remain. This result is in total agreement with the Gibbs phase rule (see [9]). In this work, we prove by the next theorem that the case $I=3$ is not reached. 
Theorem 1. Consider the maximization-problem (15)-(16), and assume that the entropy function sverifies the inequality (8).

$$
\text { Then } I<3
$$

As a consequence of Theorem 1, we notice that the van der Waals EoS, given as an example in this work, allows us only the phase change between liquid-vapor.

\subsection{Characterization of the Equilibria}

According to the Theorem 1 we consider in the sequel $I=2$ and denote the phase $I$ by index $i=1,2$. To ensure that the mass fractions $\varphi_{i}$ remain in $[0,1]$ for $i=1,2$, we assume that the specific volumes $\tau, \tau_{1}, \tau_{2}$ and the specific energies $e, e_{1}, e_{2}$ satisfy: $0<\tau_{1} \leq \tau \leq \tau_{2} ; \quad \tau_{1}<\tau_{2}, 0<e_{1} \leq e \leq e_{2} ; \quad e_{1}<e_{2} .\left(H_{1}\right)$

Using the Lagrange multipliers associate to the constraints (16) we get the equilibrium states which maximize the mixture entropy given by the maximization problem (15).

Proposition 2. Under hypothesis $(H 1)$, the equilibrium states are

1. Pure liquid or gaseous states: $\varphi_{1}=0\left(\operatorname{resp} . \varphi_{2}=0\right)$, with $\tau_{2}=\tau, \tau_{1}<\tau$ arbitrary and $e_{2}=e, e_{1}<e$ arbitrary (resp. $\tau_{1}=\tau, \tau_{2}>\tau$ arbitrary and $e_{1}=e, e_{2}>e$ arbitrary).

2. Coexistence states: $\varphi_{1} \varphi_{2} \neq 0$, then $(H 2)$ holds with $\left(\tau_{1}, \tau_{2}, e_{1}, e_{2}\right)$ satisfying

$$
p\left(\tau_{1}, e_{1}\right)=p\left(\tau_{2}, e_{2}\right), \quad \mu\left(\tau_{1}, e_{1}\right)=\mu\left(\tau_{2}, e_{2}\right), \quad T\left(\tau_{1}, e_{1}\right)=T\left(\tau_{2}, e_{2}\right)
$$

Thus, at the thermodynamic equilibrium, when the two phases are present, the system is characterized by the equality of pressures, chemical potentials and temperatures.

\section{Conclusion}

In this work, we presented a mathematical reason for the Gibbs phase rule based on convex analysis and we present the reason on which an EoS like the van der Waals EoS for example cannot be used to describe the phase transition between more than two phases. It will be interesting firstly to create a dynamical model allowing the capture of the equilibrium states. Another perspective is to use the latter as a relaxation term to the equilibrium for the Euler equations authorizing the appearance of the phase change as it is done in [6].

\section{References}

[1] G. Faccanoni, S. Kokh, and G. Allaire, "Modelling and simulation of liquid-vapor phase transition in compressible flows based on thermodynamical equilibrium," ESAIM Math. Model. Numer. Anal., vol. 46, no. 5, pp. 1029-1054, 2012.

[2] P. Helluy and N. Seguin, "Relaxation models of phase transition flows," ESAIM: Mathematical Modelling and Numerical Analysis, vol. 40, no. 2, pp. 331-352, 2006.

[3] H. B. Callen, Thermodynamics and an introduction to thermostatistics, 2nd ed. Wiley and Sons, 1985.

[4] J. W. Gibbs, The Collected Works of J. Willard Gibbs, vol I: Thermodynamics. Yale University Press, 1948.

[5] G. Faccanoni, "Etude d'un modèle fin de changement de phase liquide-vapeur. Contribution à l'étude de la crise d'ébullition," Ph.D. Thesis, Ecole Polytechnique X, 2008.

[6] F. James and H. Mathis, "A relaxation model for liquid-vapor phase change with metastability," Commun. Math. Sci., vol. 14, no. 8, pp. 2179-2214, 2016.

[7] J.-B. Hiriart-Urruty, Optimisation et analyse convexe. EDP sciences, 2012.

[8] R. T. Rockafellar, "Convex analysis," Princeton Landmarks in Mathematics. Princeton University Press, Princeton, NJ, 1997.

[9] R. G. Mortimer, Physical chemistry, 3rd ed. 2008. 